\title{
Model Calculations of Near Solar Dust Properties
}

\author{
I. Mann and H. Ishimoto \\ Max-Planck-Institut für Aeronomie, Postfach 20, D-37189 \\ Katlenburg-Lindau, Germany \\ H. Okamoto and T. Mukai \\ Department of Earth and Planetary Sciences, Faculty of Science, Kobe \\ University, Nada 657, Kobe, Japan
}

\begin{abstract}
We discuss efforts to explain the dust distribution, as it was derived from the $\mathrm{F}$-coronal brightness, mainly how to explain a constant number density near the Sun. It is shown that silicate particles have temperatures below the blackbody temperature if the impurity from absorbing material amounts to less than $0.1 \%$ in volume. This effect is especially significant for porous particles and may explain why particies can approach to less than $3 \mathrm{R}_{\odot}$ from the Sun. Another effect may arise from particles on high eccentricity orbits and from the influence of collisional fragments. Whereas on the one hand heat resistant silicate particles may cause a stable dust cloud in the solar vicinity, on the other hand dynamical effects may cause a variation of the number density in the corona in short time scales.
\end{abstract}

\section{Introduction}

Present knowledge about interplanetary dust particles around the Sun is somewhat limited, being mainly based on remote sensing. Recent observational data (Mann \& MacQueen 1993) can be fitted with a constant number density of particles within a solar distance of less than 3 to 9 solar radii $\left(R_{\odot}\right)$. A fit to the data with number density proportional to $\mathrm{r}^{-0.4}$ is still possible, but the slope is clearly flatter than in the Zodiacal light. The data were taken within minutes during an eclipse; however, there is no evidence for strong temporal variation, since the images cover the whole corona and show no local structures. The Poynting-Robertson drift of dust particles in circular orbits results in an increase of number density proportional to $r^{-1}$ (where $r$ denotes the distance from the Sun). Zodiacal light observations (Levasseur-Regourd et al. 1991) tend to indicate a slightly steeper increase towards the Sun. However they include the variation of both, number density and optical properties. The constant number density from solar distances of 3 to $9 \mathrm{R}_{\odot}$, indicates a zone of diminished particle number. In addition to this flat distribution we also have to understand how particles can approach regions as close as $3 \mathrm{R}_{\odot}$ to the Sun since, usually, a dust free zone was assumed to start at about 4 or $5 R_{\odot}$. 


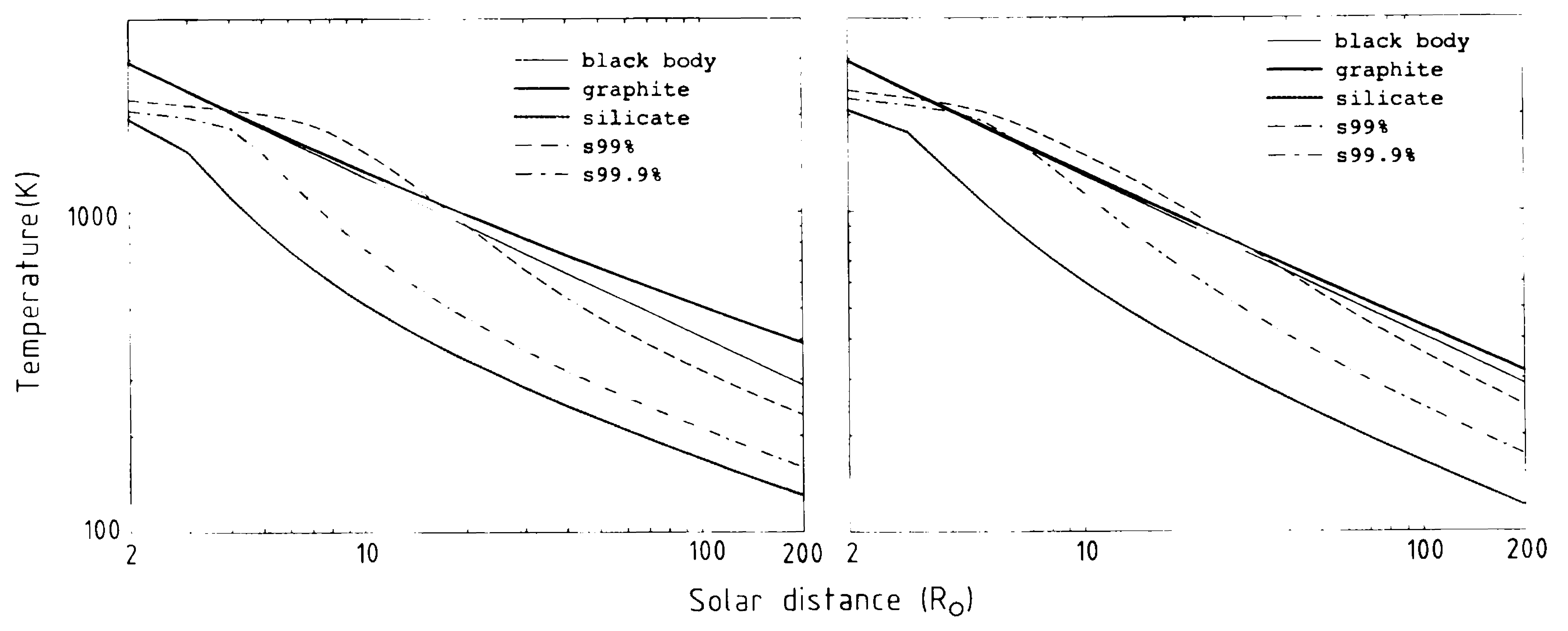

Figure 1. Temperature of two component particles of $10 \mu \mathrm{m}$ effective radius with $\mathrm{D}=2.98$ vs solar distance $\mathrm{r}$. The volume fraction of graphite is $0,0.1 \%$, and $1 \%$, the solid line gives the blackbody temperature (from Mann et al. 1994). The left part of the figure shows the profile for fractal aggregates the right part the profiles for spherical particles.

\section{Temperature Profiles and Sublimation}

To describe irregular particles we apply the fractal aggregate model (e.g. Kozasa et al. 1992 and references therein). A three-dimensional Monte-Carlo simulations for grain-grain collisions, has been performed using a similar method to that described in Mukai et al. (1992) and Kozasa et al. (1992). We calculate so-called ballistic particle-cluster aggregates (BPCA) of a fractal dimension $\mathrm{D}=2.98$ (i.e. porosity about 0.85 ) with two different types of constituent particles, i.e. dielectric and absorbing particles. The temperature $\mathrm{T}$ of particles is attained by the balance between input and output energy using Mie and Maxwell-Garnett theory, as described elsewhere (Mann et al. 1994). We note that the procedure of the grain model production bears no direct relation to the dust formation and composition in interplanetary space but is just an analog description.

Our computed results of $T(R)$ are illustrated for aggregates with $r_{\text {eff }}=$ $10 \mu \mathrm{m}$ and $\mathrm{D}=2.98$ and for a compact sphere with the same radius (Fig. 1). In general the temperature of particles increases with decreasing solar distance and the beginning of sublimation at temperatures close to $1800 \mathrm{~K}$ gives a point of inflection towards a smaller increase of the temperature profile. As far as the sublimation is concerned, it arises at a distance $r<3 R_{\odot}$ from the Sun for the pure silicate particles, in a very similar way to the case of a compact particle (see the point of inflection of each curve in Fig. 1). The distances of sublimation are listed in Table 1. It can be seen that different amounts of impurities could yield an extended zone of flat number density around the Sun. 


\section{Dynamics of Dust in High Eccentricity Orbits}

Another possibility of explaining the distribution of particles is that they approach the Sun on high eccentricity orbits and can survive in these "hotter" zones of the corona. The calculated radial slope derived for particles in hyperbolic orbits can be described as proportional to $\mathrm{r}^{-1.5}$ up to the onset of sublimation. If this starts at about $5 \mathrm{R}_{\odot}$, significant dust components can be expected up to distances of $2 R_{\odot}$ from the Sun. In this case we assume the sublimation parameters for $10 \mu \mathrm{m}$ obsidian particles which explain a dust free zone at $5 \mathrm{R}_{\odot}$ in a "traditional" model. The superposition of these two components, one in circular orbits and one in hyperbolic orbits, with additional components of particles in high eccentricity orbits may cause a flat distribution of dust in the corona.

If we assume mutual collision of dust particles it is possible to produce particles that are injected into the "dust-free zone". We can estimate from laboratory measurements (Fujiwara et al. 1977), that fragments of $10 \mu \mathrm{m}$ size can survive a catastrophic collision of zodiacal dust particles of $10 \mu \mathrm{m}$, respectively $100 \mu \mathrm{m}$ size. By taking average values of the mean speed of the dust particles and different values $f$, which denotes the energy transfer from the kinetic energy to the maximum fragment we can calculate the possible new velocities of the fragments and hence the distribution of new orbits. The total kinetic energy of impact ejecta depends strongly on the material of the target and the projectile and also the unknown parameter, mass by fraction of the energy which goes into the largest fragment. Hence we applied three different values of $f(f=0.1,0.01$, $0.001)$ and calculated the orbits of the largest fragments produced between 5 and $50 \mathrm{R}_{\odot}$, two of the calculations are presented in figure 2 which shows the perihelion distances $q$ and eccentricities e of orbits for particles produced by mutual collisions. The orbits are those for the largest produced fragment for different fractions of the kinetic energy going into these fragments. It can be seen that in all cases there is a significant amount of the particles injected into the dust free zone. However, the fragments are small and cannot make up the total F-corona brightness.

Table 1. Sublimation of $10 \mu \mathrm{m}$ sized particles. Comparison of a compact particle with an aggregate of fractal dimension $\mathrm{D}=2.98$.

\begin{tabular}{|c|c|c|}
\hline \multirow{2}{*}{$\begin{array}{l}\text { Volume fraction } \\
\text { of carbon } \\
(\%)\end{array}$} & \multicolumn{2}{|c|}{ Distance of sublimation $\left(\mathrm{R}_{\odot}\right)$} \\
\hline & Fluffy & Compact \\
\hline 0(pure silicate) & $2-3$ & 3 \\
\hline 0.1 & 4 & 5 \\
\hline 1 & 9 & 6 \\
\hline 100 (pure carbon) & $<2$ & $<2$ \\
\hline
\end{tabular}




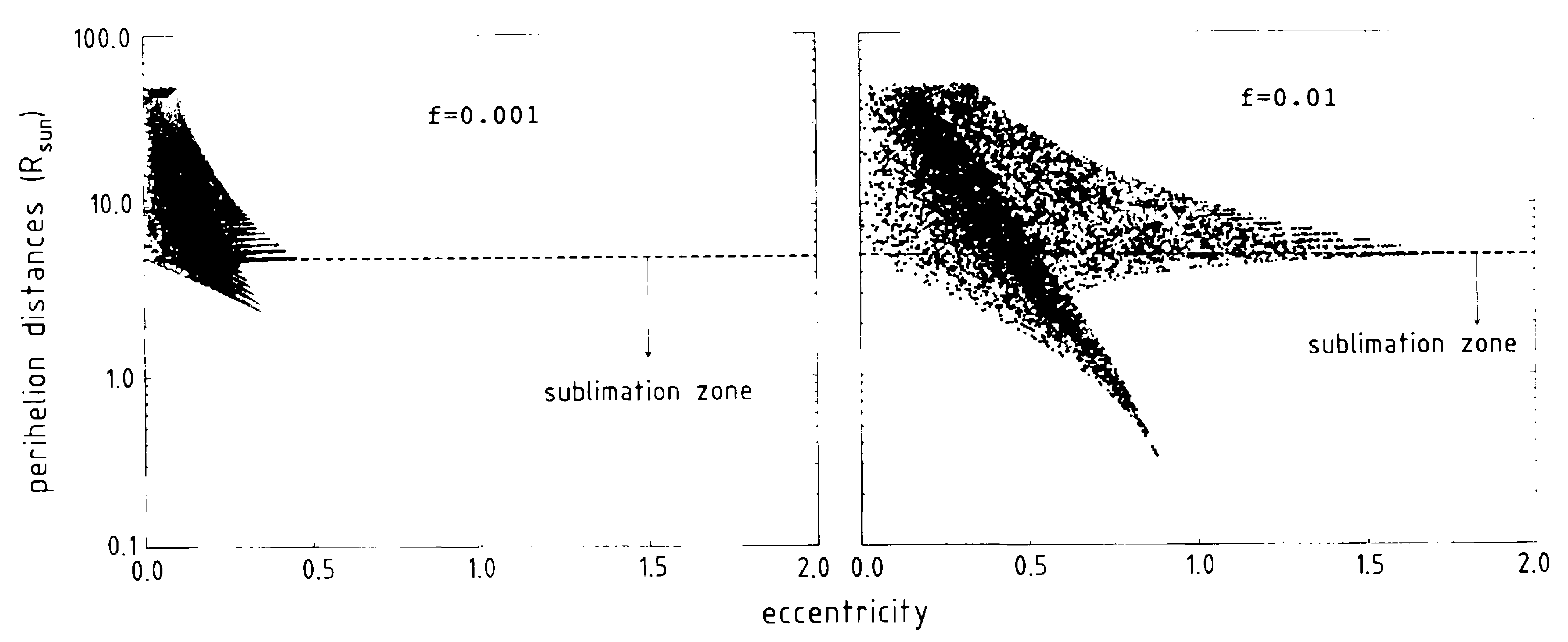

Figure 2. Perihelion distances $q$ and eccentricities e of orbits for particles produced by mutual collisions. The orbits are those for the largest produced fragment depending on the kinetic energy going into these fragments.

\section{Discussion}

We have shown that the temperature of irregulary shaped porous silicate particles may explain the existence of dust in the solar vicinity close to 2 or $3 R_{\odot}$. These particles can survive if the amount of absorbing material is very small. Moreover compact pure silicate particles of submicron size may survive in the solar vicinity. We have also shown, that different dynamical effects, can inject particles, even of $10 \mu \mathrm{m}$ size into the most inner region, but it is unlikely that they produce the total number density of particles very close to the Sun. However, it should be mentioned that such type of dynamical effects may cause a variation of the number density in the corona in short time scales.

Acknowledgments. This work was supported by the Bundesministerium für Forschung und Technologie (BMFT) (Contract 50 ON 9101).

\section{References}

Fujiwara, A., Kamimoto, G., \& Tsukamoto, A. 1977, Icarus, 31, 277

Kozasa, T., Blum, J., \& Mukai, T. 1992, A\&A, 263, 423

Levasseur-Regourd, A. C., Renard, J. B., \& Dumont, R. 1991, in Origin and Evolution of Interplanetary Dust, A. C. Levasseur-Regourd \& H. Hasegawa, Dordrecht: Kluwer, 131

Mann, I., \& MácQueen, R. M. 1993, A\&A, 275, 293

Mann, I., Okamoto, H., Mukai, T., Kimura, H., \& Kitada, Y. 1994, A\&A, 291, 1011

Mukai, T., Ishimoto, H., Kozasa, T., Blum, J., \& Greenberg, J. M. 1992, A\&A, 262,315 